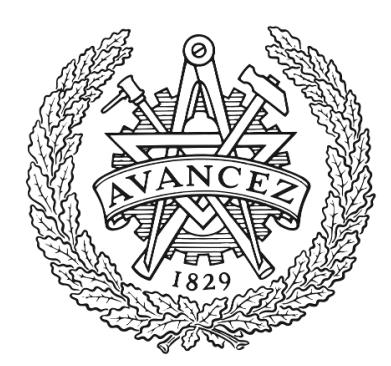

\title{
CHALMERS
}

UNIVERSITY OF TECHNOLOGY

\section{Resemblance Between Gap Waveguides and Hollow Waveguides}

Downloaded from: https://research.chalmers.se, 2023-04-26 04:53 UTC

Citation for the original published paper (version of record):

Raza Zaidi, S., Yang, J., Kildal, P. et al (2013). Resemblance Between Gap Waveguides and Hollow Waveguides. IET Microwaves, Antennas and Propagation, 7(15): 1221-1227.

http://dx.doi.org/10.1049/iet-map.2013.0178

N.B. When citing this work, cite the original published paper. 


\title{
Resemblance between Gap Waveguides and Hollow Waveguides
}

\author{
Hasan Raza, Jian Yang, Per-Simon Kildal and Esperanza Alfonso \\ Department of Signals and Systems, Chalmers University of Technology, S-41296 Gothenburg, Sweden
}

e-mail: hasan.raza@chalmers.se, jian.yang@chalmers.se,per-simon.kildal@chalmers.se, esperanza.alfonso@chalmers.se

Abstract — This paper studies the resemblance between the ridge/groove gap waveguide and the conventional ridge/hollow waveguide, respectively, by using numerical analysis. Dispersion diagrams and characteristic impedances are comparatively analyzed. Direct transition from gap waveguide to conventional waveguide and thereafter the port definitions for gap waveguides are examined in detail. The results from this study are very useful for designing, simulating and measuring gap-waveguide components of different kind.

Keyword — gap waveguide, ridge waveguide, characteristic impedance.

\section{Introduction}

Gap waveguide technology is a new way to make low-loss circuits at millimeter and sub-millimeter waves [1]-[4]. This waveguide can be realized without having any dielectric 
material inside it, and without having electrical contact between the top and the bottom metal plates, where the latter is a texture plate (periodic structure) normally in the form of metal pins with ridges or grooves in between as waveguides. This texture plate creates a high impedance surface which can be modeled ideally by a Perfect Magnetic Conductor (PMC). Therefore, propagating waves are prohibited in this parallel-plate waveguide (upper smooth metal plate and lower texture metal plate) everywhere except along the metal ridges or grooves, as long as the spacing between the upper and lower plates is smaller than a quarter wavelength. This small spacing is called as the gap, the reason for the name of this waveguide. The bandwidth of this wave prohibition is referred to as the stopband, which can be also used for packaging of both passive and active microwave circuits, realized with microstrip or co-planar waveguides, to avoid resonant modes [5],[6]. The propagation waves along the metallic ridge or groove within this periodic texture are quasi TEM mode or quasi-TE mode [7], respectively, as illustrated in Figure 1.

We will here focus our study on the gap waveguides realized by square metal pins [1], [8], but the method is also valid for other realizations of the gap waveguide [9], and the results will be reported in other publications.

The key parameters for controlling the stopband are the period of the pins, the height of the air gap between the top of the pins and the upper smooth plate (referred to as the pin gap height), and the height and width of the pins [8]. The period of the pins must be smaller than half the wavelength at center frequency, the pin gap height determines the high-end frequency of the stopband and must be smaller than quarter wavelength at center frequency, and the height and width of the pins determine the low-end frequency of the stopband [9]. 
The modes in a ridge gap waveguide propagate in the air gap between the ridge and the top plate, so ridge gap waveguide has lower ohmic loss than microstrip line does. The advantage of the gap waveguide over the conventional hollow rectangular waveguide is that the former does not require high precision manufacture at high frequency while the latter must do. The normal is that hollow waveguides and waveguide components are manufactured from two metal pieces that must be joined with good conductive metal contact, which imposes a difficulty, whereas for gap waveguides there is no such requirement since the upper and the lower plates do not join (with a air gap instead).

The purpose of this paper is to obtain the propagation characteristics of the newly-introduced ridge/groove gap waveguides by a comparative analysis to the classical ridge/rectangular waveguides [10], carried out by numerical analysis in terms of the dispersion diagrams and the characteristic impedances. Direct transition from gap waveguide to conventional waveguide and thereafter the port definitions for gap waveguides are examined in detail.

\section{Dispersion Diagram}

First, the dispersion diagrams of the ridge/rectangular waveguides and the ridge/groove gap waveguides are obtained by using the Eigenmode solver in CST Microwave Studio, where the periodic structure is assumed infinitely long in the direction of propagation. In this section, the material used for the simulation is perfect electric conductor (PEC) with zero surface roughness. The cross sectional geometries of the gap waveguide simulation models are summarized in Table 1, where the dimensions are chosen in agreement with those in [9] in order to get a targeted stopband from 10 to $20 \mathrm{GHz}$. Note that the width and the periodicity of 
the pins play significant roles to determine the stopband [9].

In Table 1, we also define the corresponding ridge/hollow waveguide with its dimensions coincidence to those of the ridge/groove gap waveguide by putting the metal side walls at the first pin's wall. Later on we will see that the propagation characteristics of the ridge/groove gap waveguides and its corresponding ridge/hollow waveguide are very similar. Therefore, we refer the corresponding ridge/hollow waveguide to as the equivalent waveguide of the gap waveguide.

The simulated dispersion diagrams of the ridge gap waveguide and its equivalent ridge waveguide are shown in Figure 2 for four different gap heights between the ridge and the top metal plate (referred to as the ridge gap height), i.e. $h=0.5,1,2,3 \mathrm{~mm}$. The pin heights and the pin gap height remain the same for all cases. We see that the stopbands of these gap waveguides are almost the same, from 11 to $22 \mathrm{GHz}$. The fundamental mode of the ridge gap waveguide with a small ridge gap height is seen to have a dispersion curve (referred to as the ridge gap dispersion) very close to that of TEM mode (the Light Line). However, the ridge gap dispersion moves away from the Light Line when the ridge gap height increases. Nevertheless, the ridge gap dispersion is very close to the dispersion curve of the fundamental mode in the equivalent ridge waveguides within the stopband.

Similarly we compare in Figure 3 the dispersion diagrams between a groove gap waveguide and its equivalent hollow rectangular waveguide. The stopband for this groove gap waveguide is from 11 to $19 \mathrm{GHz}$. The dispersion diagrams of the fundamental propagating modes in both the waveguides are similar within the stopband.

In summary, the dispersion diagram of a ridge/groove gap waveguide can be 
approximated by that of its equivalent ridge/hollow waveguide, which can be easily obtained by either analytical expressions or empirical formulas.

\section{Direct Transition between Hollow Waveguide and Gap Waveguide}

The simulations in this section were done by using both CST and HFSS, two most used commercial EM (electromagnetic) solvers, on ridge/groove gap waveguides, with the dimensions shown in Table 1 and a length of 91.5 mm (see Figure 4a).

The direct transition from ridge/groove gap waveguide to conventional ridge/hollow waveguide means that an optimal conventional ridge/hollow waveguide is chosen so that when this optimal ridge/hollow waveguide is connected directly to the ridge/groove gap waveguide, the reflection from the interface between them is the minimum, see Figure $4 \mathrm{~b}$. The direction transition is a simple and low-cost way to convert gap waveguides to conventional waveguides and therefore very useful in measurements.

By intuition and also proven with a parametric study, the optimal ridge/hollow waveguide for the direct transition is the equivalent waveguide of the gap waveguide. However, the location of the interface for the direct transition should be treated with care. A parametric study on $k_{1}$ which defines the location of the interface (see Figure 5) has been carried out. The best result appears when $k_{1}=p / 2$, where $p$ is the spacing between the walls of the pins, as shown in Figure 6.

Then, using the direct transition as a reference, the equivalent waveguide port for gap waveguide in CST and HFSS is defined, which makes the simulation in both solvers more efficient. The port has the same dimensions of the equivalent waveguide with electric shielding (corresponding to a zero-length equivalent waveguide) and is located at the 
interface $p / 2$ away from the wall of the first pins, as shown in Figure 5. Note that the results provided by CST and HFSS are very similar.

Similarly, the equivalent waveguide port for groove gap waveguide is defined to coincide with the equivalent hollow waveguide, shown in Figure $4 \mathrm{~b}$, after a parametric study.

Figure 7 and Figure 8 show the S-parameters for the two cases of the equivelant waveguide port shown in Figure 4a, with the ridge gap waveguides of different ridge gap heights and the groove gap waveguide in Table 1 , respectively. We see that the reflection coefficient $\mathrm{S}_{11}$ by using the equivalent waveguide port is below $-35 \mathrm{~dB}$ over most of the stopband if the ridge gap height is smaller than or equal to $1 \mathrm{~mm}$ but increases when the ridge gap height increases. For the groove gap waveguide, $\mathrm{S}_{11}$ is below $-30 \mathrm{~dB}$ over most of the stopband, except in the beginning of the stopband. Thus, the matching between the gap waveguide and the equivalent waveguide port is good except at the low-end of the stopband. The periodic nature of the reflection coefficient comes from the two interfering reflections, from the ports at both ends of the waveguides. This means that the reflection coefficient from each port interface is $6 \mathrm{~dB}$ lower than the peaks of the combined reflection coefficient $\mathrm{S}_{11}$ $[11]$.

The simulation models include also the ohmic loss by using the lossy copper $(\sigma=5.8 \times$ $10^{7} \mathrm{~S} / \mathrm{M}$ ) in the models. The transmission coefficient $\mathrm{S}_{21}$ for the $91.5 \mathrm{~mm}$ long gap waveguide is about $-0.1 \mathrm{~dB}$ within the part of the stopband where the reflection coefficient is low.

\section{Characteristic Impedance}

The characteristic impedance of the ridge gap waveguide is determined mainly by the ridge width and the ridge gap height [12]. There were several methods available to calculate the 
approximate value of the characteristic impedance of ridge gap waveguide [13]-[14]. In this section, we introduce a new numerical method to have an accurate calculation of the characteristic impedance for a ridge gap waveguide.

We know from the previous section that the reflection coefficient of the direct transition is below $-30 \mathrm{~dB}$. This indicates that the equivalent ridge waveguide is well matched to the ridge gap waveguide, and we can use, as the first approximation, the simulated characteristic impedance of the equivalent ridge waveguide as that of the ridge gap waveguide. Then, we apply a correction to the first-order approximated characteristic impedance by using the simulated reflection coefficient of the 2-port network shown in Figure 4a, the gap waveguide under test with its equivalent waveguide ports at the two ends. The reflection coefficient $r$ of one transition can be written as

$$
\mathrm{r}=\frac{Z_{o}-Z_{\text {port }}}{Z_{o}+Z_{\text {port }}}
$$

where $Z_{\text {port }}$ is the characteristic impedance of the equivalent ridge waveguide, and $Z_{o}$ is the characteristic impedance of the ridge gap waveguide. The S-parameter $S_{11}$ of the 2-port network in Figure 4 can be estimated by using the theory of small reflections in [11] as long as $|r|<0.2$ :

$$
S_{11} \approx \mathrm{r}\left(1-e^{-j 2 \beta l}\right)
$$

where $l$ is the length of the ridge gap waveguide. Using (1) and (2), we have

$$
Z_{o} \approx \frac{\left(1-e^{-j 2 \beta l}\right)+S_{11}}{\left(1-e^{-j 2 \beta l}\right)-S_{11}} Z_{\text {port }}
$$

We simplify the above calculation by i) calculating the values of $Z_{o}$ at the frequencies $f_{i}$ where $S_{11}$ has its peaks, by

$$
Z_{o}\left(f_{i}\right) \approx \frac{2+S_{11 \text { peak }}\left(f_{i}\right)}{2-S_{11 \text { peak }}\left(f_{i}\right)} Z_{\text {port }}
$$


ii) the impedance curves over the whole band is obtained by interpolation and extrapolation. Figure 9 shows the impedance calculation for ridge gap waveguides with different ridge gap heights while the ridge width is kept the same in all cases.

From Figure 9, it is observed that the discrepancy between the gap waveguide impedance $Z_{o}$ and the port impedance $Z_{\text {port }}$ (the approximation of $Z_{o}$ ) increases with the increase of the ridge gap height, especially at the low end of the stopband. Re-examining Figure 2, it can be observed that the difference between the dispersion diagrams of the fundamental modes of both the ridge waveguide and ridge gap waveguide increases with the increase of the ridge gap height, especially at the low end of the stopband. This explains this impedance discrepancy.

The same procedure is also applied to groove gap waveguide, where the wave impedance for the waveguide is calculated through the equivalent waveguide port impedance $Z_{\text {port }}$ and the S-parameter $S_{11}$. The results are shown in Figure 10 .

The characteristic impedance of the ridge gap waveguide was previously calculated by several methods, such as the half-stripline-model method in [13], the circuit model $V / I$ and $P / I^{2}$ method in [14]. Figure 11 compares the present method to those previous methods on the characteristic impedance for the ridge gap waveguide with the dimensions shown in the figure. Table 1 lists, for clarification purpose, some digit values of them obtained by these different methods at three frequencies. We believe that the present method has a better accuracy.

\section{Conclusions}

The dispersion diagram and the characteristic impedance of the newly-introduced gap 
waveguide can be determined by its equivalent conventional waveguide with a proper correction, concluded by the comparative study presented in the paper. A new accurate method to determine the characteristic impedance of a gap waveguide has been introduced and compared to the previous methods. The simple direct transition between gap waveguide and conventional waveguide proposed in the paper can provide an efficient transition for gap waveguide measurements and numerical port definition. The results of this study are very useful for designing, simulating and measuring gap-waveguide components

\section{Acknowledgement}

This work has been supported by The Swedish Foundation for Strategic Research (SSF) within the Strategic Research Center CHARMANT and Pakistan's NESCOM scholarship program. 


\section{References:}

[1] Kildal, P.-S., Alfonso E., Valero-Nogueira, A., and Rajo-Iglesias, E.: 'Local Metamaterial-Based Waveguides in Gaps Between Parallel Metal Plates', IEEE Antennas and Wireless Propagation Letters, 2009, (8), pp. 84 - 87.

[2] Raza, H., Yang, J.: 'Compact UWB power divider packaged by using gap waveguide technology', Proceedings of 6th European Conference on Antennas and Propagation, EuCAP 2012. Prague, 26-30 March 2012, pp. 2938-2942.

[3] Raza, H., Yang, J.: 'A low loss rat race balun in gap waveguide technology’, Proceedings of the 5th European Conference on Antennas and Propagation, EUCAP 2011. Rome, 11-15 April 2011, pp. 1230-1232.

[4] Yang, J., Raza, H.: 'Empirical formulas for designing gap-waveguide hybrid ring coupler', submitted to IEEE Microwave and Wireless Components Letters, 2012.

[5] Rajo-Iglesias, E., Uz Zaman, A., Kildal, P.-S.: 'Parallel plate cavity mode suppression in microstrip circuit packages using a lid of nails', IEEE Microwave and Wireless Components Letters, Vol. 20, No. 1, pp. 31-33, Dec. 2009.

[6] Uz Zaman, A., Yang, J., Kildal, P.-S.: 'Using lid of pins for packaging of microstrip board for descrambling the ports of Eleven antenna for radio telescope applications', 2010 IEEE International Symposium on Antennas and Propagation, Toronto, Canada, July, 2010.

[7] Kildal, P.-S.: 'Three metamaterial-based gap waveguides between parallel metal plates for mm/submm waves', 3rd European Conference on Antennas and Propagation, 2009. EuCAP 2009. Berlin, Germany, 23-27 March 2009.

[8] Kildal, P.-S., Uz Zaman, A., Rajo, E., Alfonso, E., Valero Nogueira, A.: 'Design and 
Experimental Verification of Ridge Gap Waveguide in Bed of Nails for Parallel Plate Mode Suppression', IET Microwave Antennas and Propag., Vol. 5, No. 3, pp. 262-270, March 2011.

[9] Rajo-Iglesias, E., Kildal, P.-S.: 'Numerical studies of bandwidth of parallel plate cut-off realized by bed of nails, corrugations and mushroom-type EBG for use in gap waveguides', IET Microwaves, Antennas \& Propagation, Vol. 5, No 3, pp. 282-289, March 2011.

[10] Cohn, S. B.: 'Properties of ridge waveguide', Proc. IRE, vol. 35, pp. 783-788, August, 1947.

[11] Kildal, P.-S.: 'Foundations of Antennas - A Unified Approach', 2nd Edition, p. 70, Studentlitteratur, 2000.

[12] Polemi, A., Maci, S.: 'Closed form expressions for the modal dispersion equations and for the characteristic impedance of a metamaterial-based gap waveguide', IET Microw. Antennas Propag., 2010, Vol. 4, Iss. 8, pp. 1073-1080.

[13] Alfonso, E., Kildal, P.-S., Valero-Nogueira, A., Baquero, M.: 'Study of the characteristic impedance of a ridge gap waveguide', IEEE Antennas and Propagation Society International Symposium APSURSI’09, pp. 1-4, June 2009.

[14] Alfonso, E., Baquero, M., Valero-Nogueira, A., Herranz, J.I., Kildal, P.-S.: 'Power divider in ridge gap waveguide technology', 4th European Conference on Antennas and Propagation (EuCAP 2010), Barcelona, Spain, 12-16 April 2010.

[15] Pozar, D.: ‘Microwave Engineering’, 3rd Edition, p. 139, Wiley, 2005. 


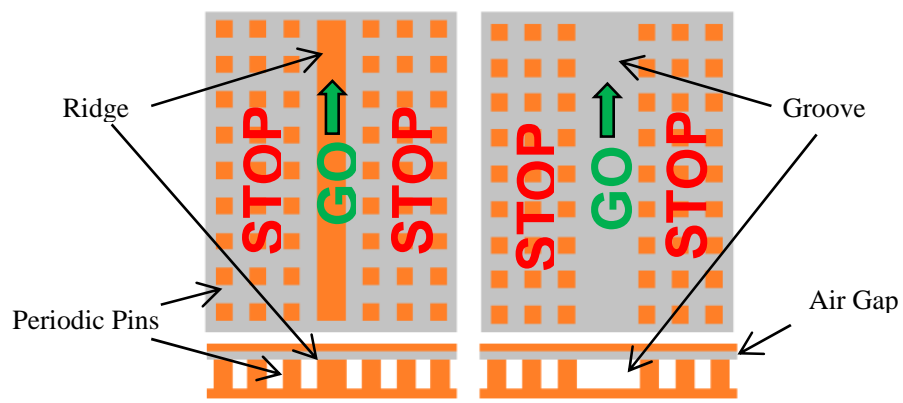

Figure 1 Top (upper) and front (lower) view of basic structures of ridge gap waveguide (left) and groove gap waveguide (right). The grey region is the air gap and the terms STOP and GO are used to depict the stop region and propagation region, respectively. 


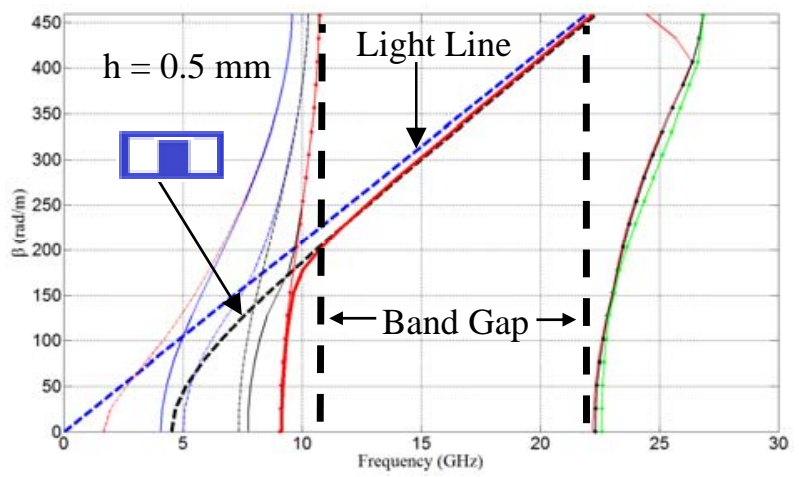

(a)

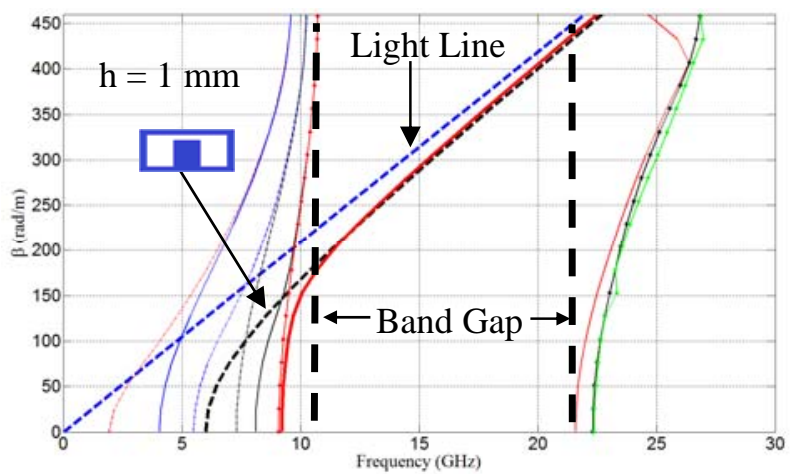

(b)

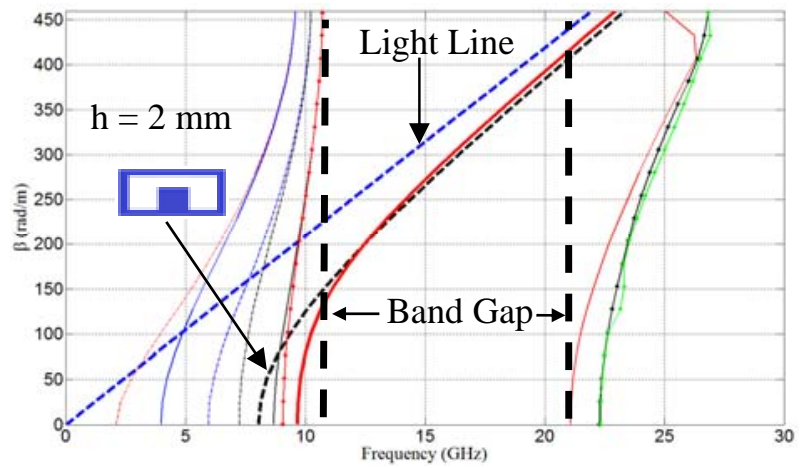

(c)

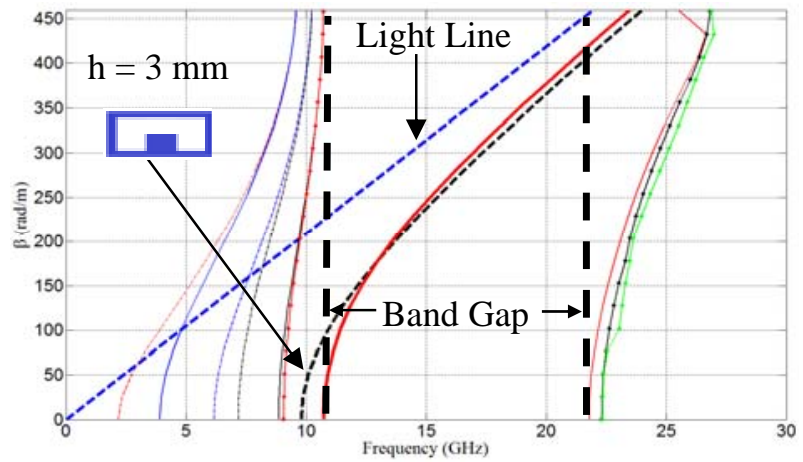

(d)

Figure 2 Dispersion diagram of ridge gap waveguide with different ridge gap heights. The size of the pins and the pin gap heights are the same for all cases. The two black vertical lines mark the beginning and end of the stopband, and the dashed black line is the dispersion diagram of the equivalent hollow ridge waveguide. 


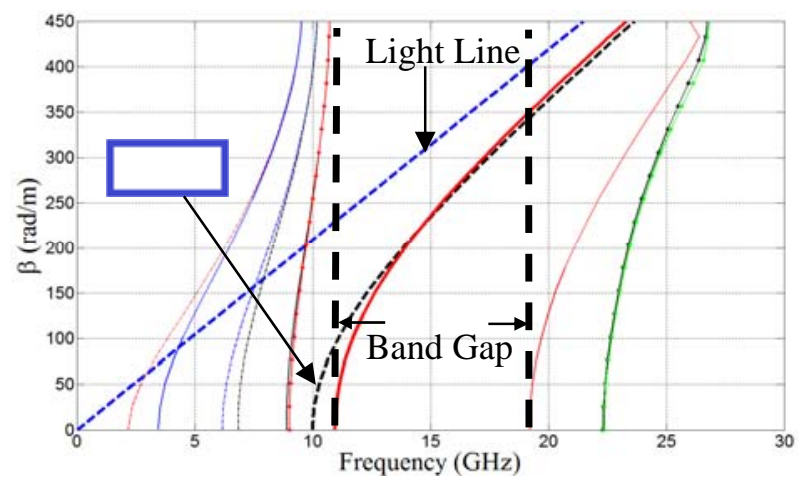

Figure 3 Dispersion diagrams of groove gap waveguide and equivalent rectangular waveguide (dashed black line). The two black vertical lines mark the beginning and end of the stopband.
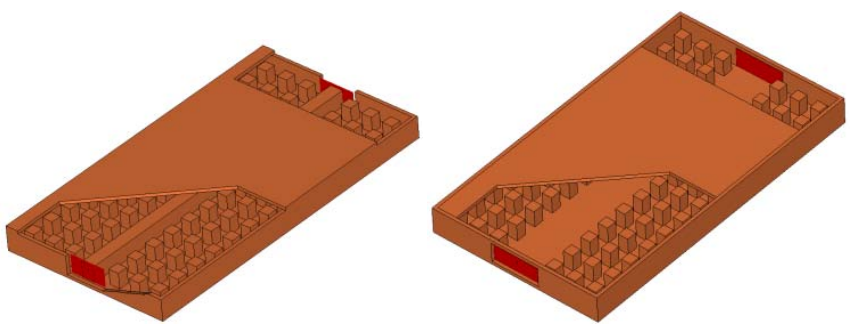

(a)
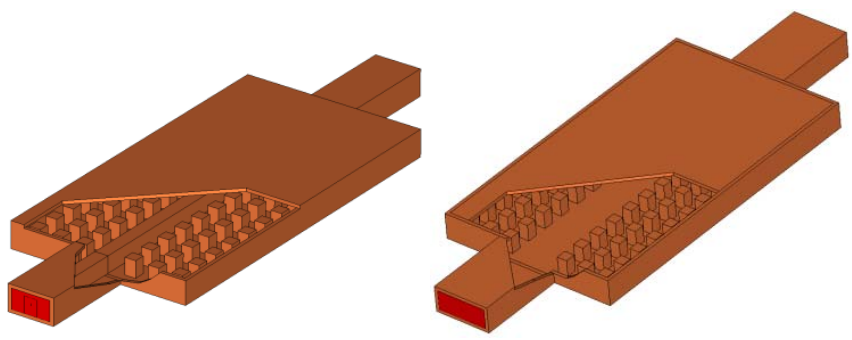

(b)

Figure 4 Port configuration for calculating the S-parameters of the gap waveguides. (a) Ports directly attached to ridge/groove gap waveguide. (b) Ports attached to the equivalent hollow waveguide transitions.

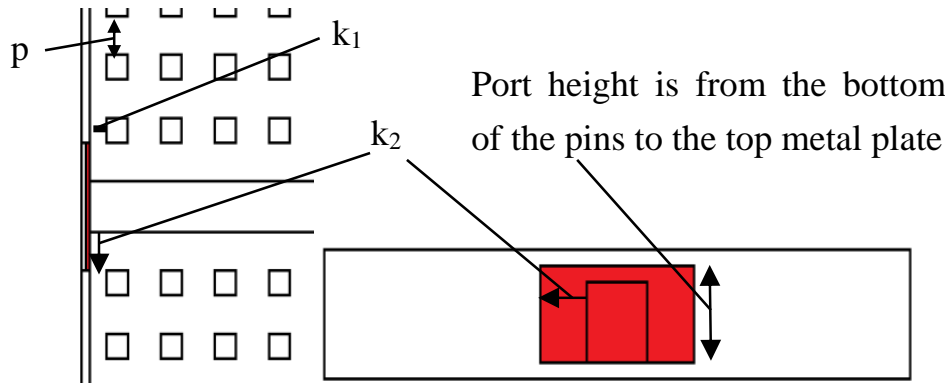

Figure 5 Illustration of the parametric study of the port location and width. 


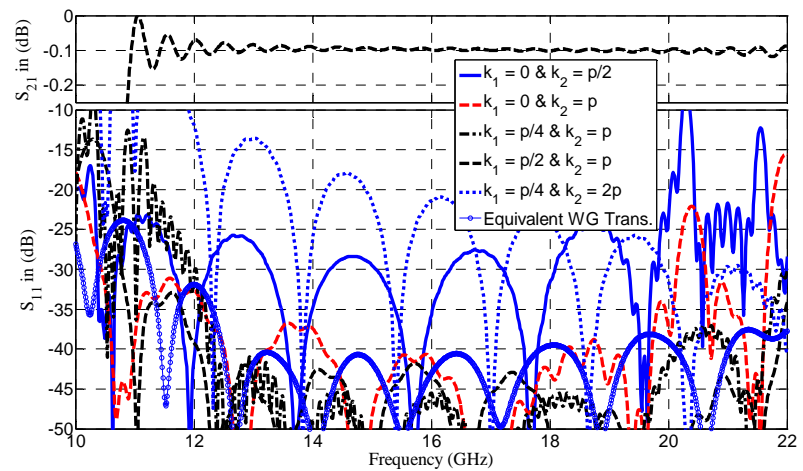

(a)

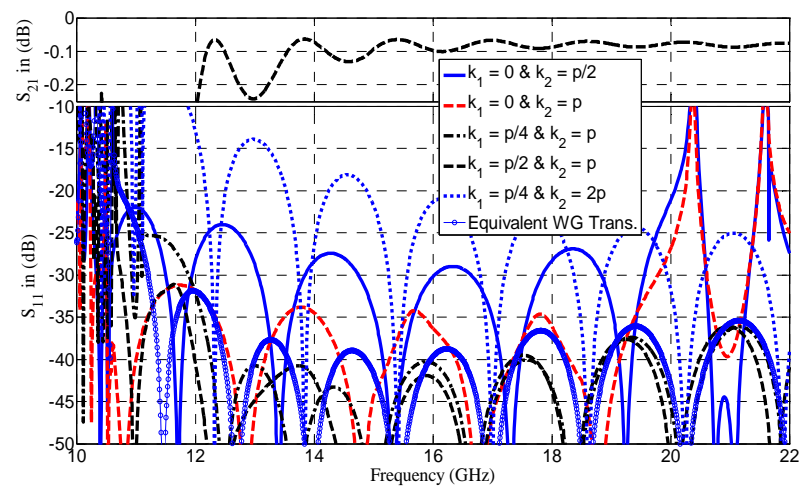

(b)

Figure 6 S-parameters at different port locations and width, using CST (a) and HFSS (b) 


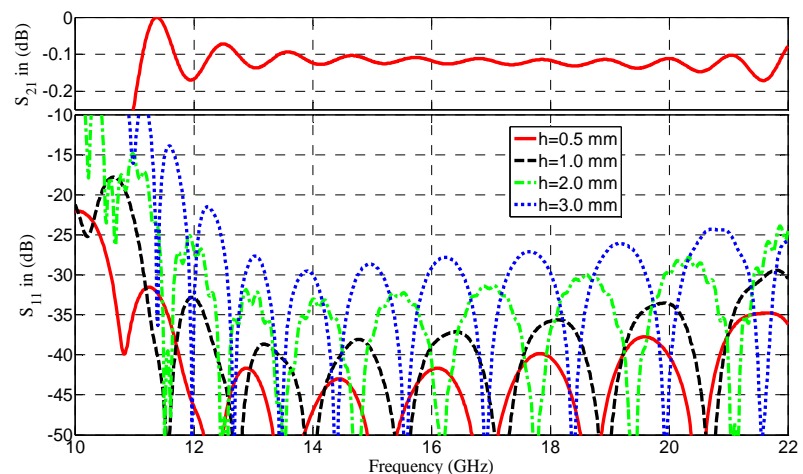

(a)

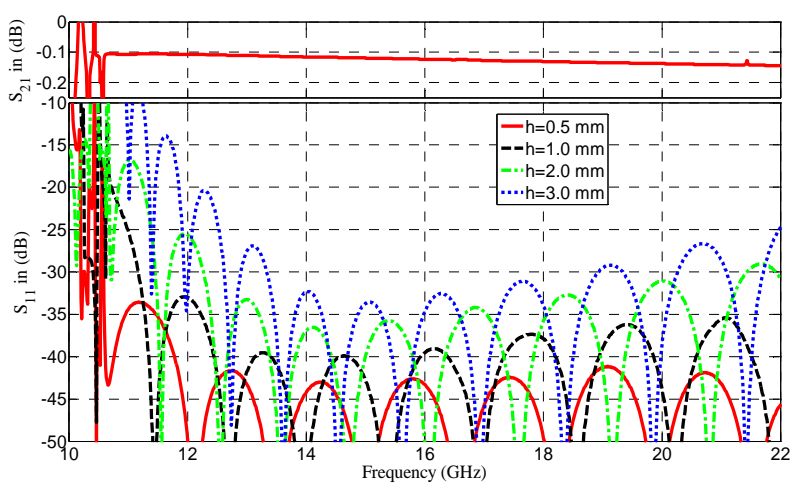

(b)

Figure 7 S-parameters of the ridge gap waveguide for different ridge gap heights, using CST (a) and HFSS (b).

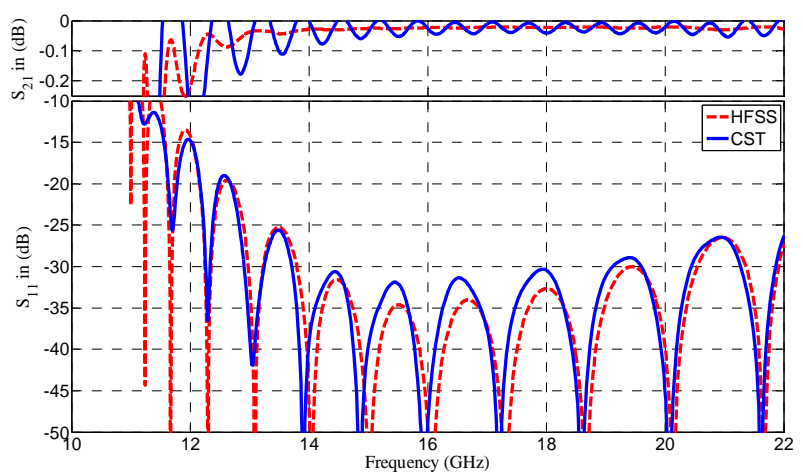

Figure 8 S-parameters of the groove gap waveguide using both CST and HFSS. 


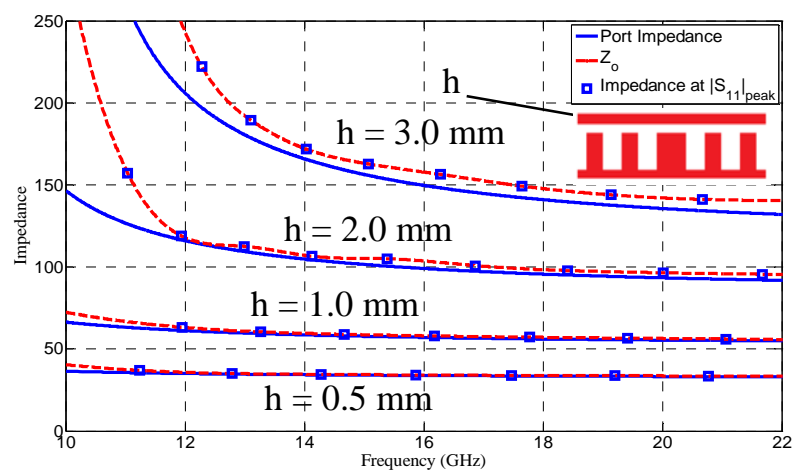

Figure 9 Characteristic impedance of ridge gap waveguide from HFSS port model and our corrected result $\left(Z_{0}\right)$.

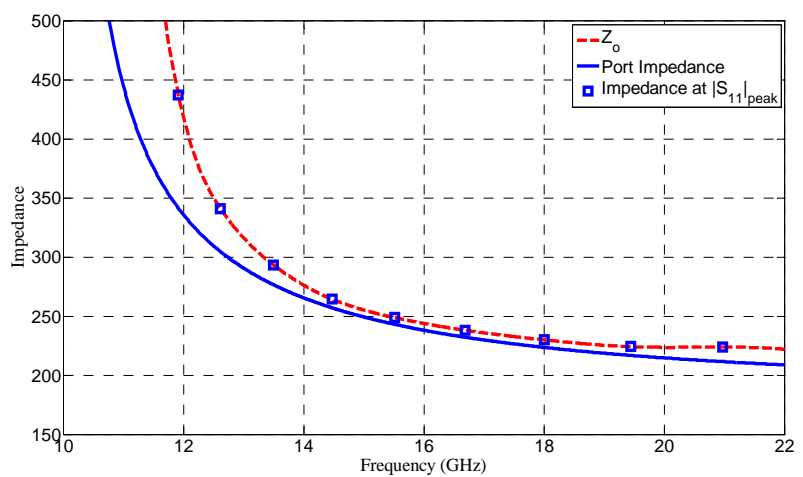

Figure 10 Wave impedance in groove gap waveguide from HFSS port model and our corrected result $\left(\mathrm{Z}_{0}\right)$.

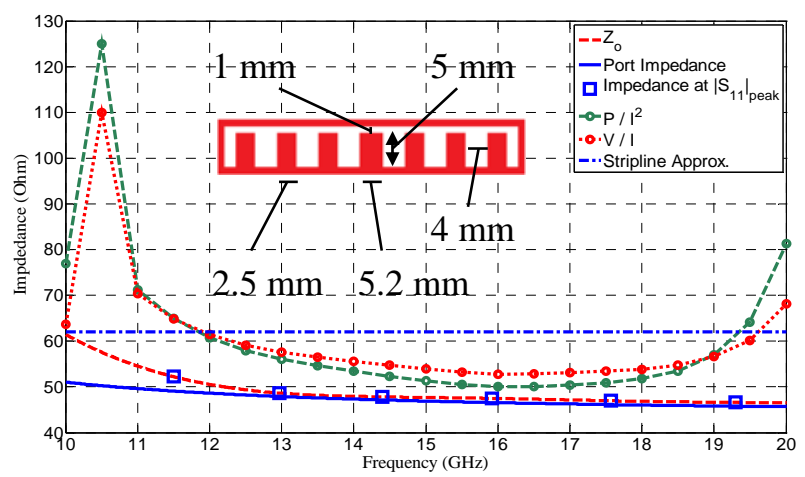

Figure 11 Comparison of the characteristic impedance of the ridge gap waveguide by using the present method, the stripline model method in [13] and the circuit model V/I and P/ ${ }^{2}$ method in [14]. 
Table 1 Cross-sections with dimensions of the simulated ridge and groove gap waveguides in Figure 1 (left), and of the equivalent hollow ridge and rectangular waveguides (right).

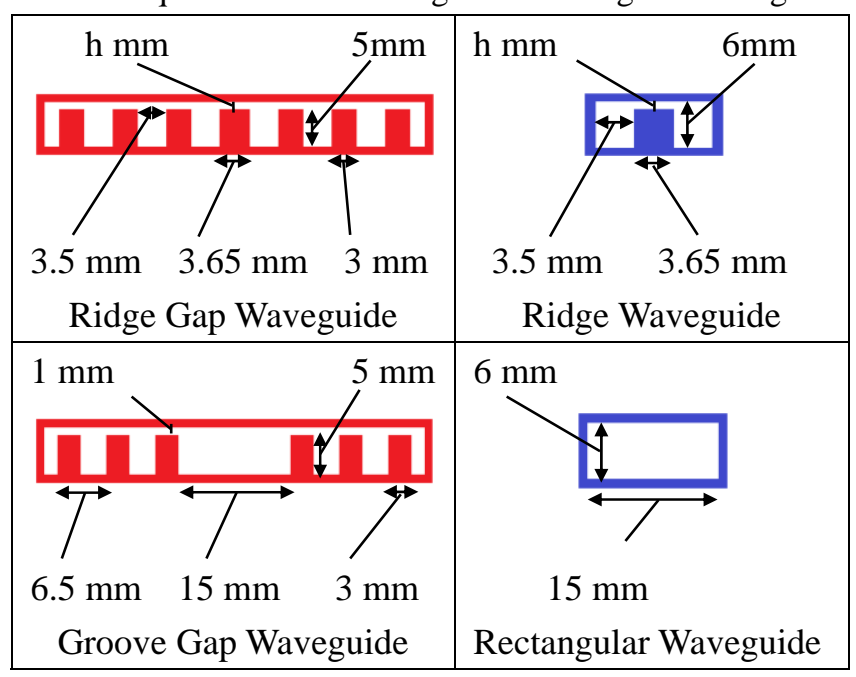

Table 1 Characteristic impedance in Ohm of the ridge gap waveguide in Figure 11, obtained by different methods at three frequencies.

\begin{tabular}{|c|c|c|c|}
\hline Characteristic impedance (Ohm) & $12 \mathrm{GHz}$ & $15 \mathrm{GHz}$ & $18 \mathrm{GHz}$ \\
\hline$P / I^{2}$ method [14] & 57.5 & 50.2 & 47.9 \\
\hline$V / I$ method [14] & 60.0 & 53.5 & 51.9 \\
\hline Half-stripline model method [13] & 62.0 & 62.0 & 62.0 \\
\hline Port-impedance method in the paper & 48.6 & 46.9 & 46.0 \\
\hline Port-impedance-with-correction method in the paper & 50.5 & 47.7 & 46.8 \\
\hline
\end{tabular}

